\title{
La convivencia escolar en un grupo de 4to. Grado de educación primaria como parte de la formación cívica y ética
}

DOI: $10.46932 / \mathrm{sfjdv} 2 \mathrm{n} 1-051$

Received in: November 1st, 2020

Accepted in: December 30th, 2020

\author{
Lic. Jorge Luis García Pérez \\ Escuela Normal Miguel F. Martínez \\ Av. Constitución s/n Col. Centro. Monterrey, Nuevo León. \\ E-mail: jorge.garcia@al.enmfm.edu.mx \\ Dra. Edith Arévalo Vázquez \\ Escuela Normal Miguel F. Martínez \\ Av. Constitución s/n Col. Centro. Monterrey, Nuevo León. \\ E-mail: edith.arevalo@enmfm.edu.mx
}

\section{RESUMEN}

Se presenta un estudio cuyo objetivo fue favorecer la convivencia escolar en un grupo de 4to. grado de educación primaria, mediante un proyecto de intervención a desarrollarse desde la asignatura de Formación Cívica y Ética. Es un estudio con enfoque cualitativo y una metodología de investigaciónacción, al valorar la enseñanza como una actividad reflexiva que se orienta a mejorar la práctica de los profesores en las aulas. Los participantes son 27 alumnos y un estudiante normalista de octavo semestre que realiza su práctica profesional en una escuela primaria en Nuevo León. A través del proyecto de intervención se buscó atender el tema de la convivencia entre los alumnos, con el ánimo de generar mejores ambientes de cooperación y armonía, necesarios para propiciar un clima de confianza en el aula $\mathrm{y}$, en consecuencia, de aprendizaje. El estudio ha posibilitado entender que los valores no se han perdido, ahí están, ya que cada persona tiene valores apropiados en función de la importancia y el significado que éstos les asignen en su escala de valores.

Palabras clave: Convivencia escolar, valores, formación cívica y ética.

\section{PLANTEAMIENTO DEL PROBLEMA}

La participación en las jornadas de práctica docente realizadas en el transcurso de la formación inicial recibida en la Escuela Normal ha posibilitado identificar que regularmente la convivencia en las aulas suele ser tratada de manera rígida, buscando un control sobre los alumnos más que un fomento a la convivencia. Como resultado, no se desarrolla un ambiente de aprendizaje en donde se trabajen los conocimientos, actitudes y valores necesarios para que los alumnos aprendan a ser y a convivir; pese a que sean dos de los propósitos que postulan desde el Informe Delors (1996).

Asimismo, en el grupo de 4to. grado donde un estudiante normalista de octavo semestre de la Licenciatura en Educación Primaria realiza la práctica profesional, ha vivido cómo diariamente se generan situaciones conflictivas entre los alumnos. Sin embargo, ante estos escenarios se han realizado limitadas 
actividades o estrategias didácticas con la intención de evitar situaciones conflictivas entre los alumnos día con día.

La necesidad de ahondar en el tema de la convivencia escolar radica, además, en que el Plan de Estudios 2011 bajo su visión humanista plantea en el enfoque del campo de formación Desarrollo Personal y para la Convivencia la existencia de dos ejes articuladores en el campo la conciencia del sí y la convivencia que destacan la necesidad de "crear ambientes en donde los aspectos afectivos (emociones y sentimientos), se encuentren íntimamente relacionados con el pensamiento, la comprensión y los procesos conscientes de metacognición y autorregulación que intervienen en el aprendizaje” (SEP, 2011, p. 433). Asimismo, es necesario propiciar un ambiente de aprendizaje que promueva las relaciones democráticas y el apego a los valores universales que la convivencia necesita para ser pacífica y armónica. Por lo que si no se desarrolla un ambiente de aprendizaje que favorezca la convivencia, ¿realmente se está cumpliendo con lo propuesto desde el Plan de Estudios 2011?

Por su parte, el Instituto Nacional para la Evaluación de la Educación (INEE) en su informe del 2018, titulado La Educación Obligatoria en México, destaca la importancia de prestar atención a la convivencia escolar a través de su capítulo "Los ambientes de enseñanza y aprendizaje: la convivencia escolar" (INEE, 2018). En este apartado incluye el análisis de resultados respecto a este relevante tema, tanto en educación primaria como en educación secundaria.

En este sentido, las estrategias que el docente lleve a cabo para atender este relevante tema deben resultar significativas para sus estudiantes. Sin duda, el docente es pieza clave para la generación de mejores ambientes para la convivencia y el aprendizaje. Razón por la que el presente estudio ha centrado la atención en esta problemática, surgiendo como pregunta inicial ¿Qué resultados ofrece implementar un proyecto de intervención centrado en atender la convivencia escolar en un grupo de 4to. grado de educación primaria, mediante la asignatura de Formación Cívica y Ética? Y como objetivo general para el estudio se espera analizar la forma en que la implementación de un proyecto de intervención favorece la convivencia en un grupo de 4to. grado de educación primaria mediante el tratamiento de la asignatura de Formación Cívica y Ética.

\section{MARCO TEÓRICO}

El Plan y Programas de Estudios (bien sea el 2011 o 2018), es el documento rector de la educación mexicana hoy en día. En él están definidos el perfil de egreso, las competencias, propósitos, estándares curriculares y enfoques de las disciplinas que se imparten en el Sistema Educativo Mexicano, así como los aprendizajes esperados a favorecer en cada grado escolar. Marcan la pauta sobre cómo se espera sea la educación mexicana con el propósito de lograr una educación de calidad en el país; esperando atender, 
asimismo, el plan educativo que se propone desde la Organización para la Cooperación y Desarrollo Económico (OCDE).

Por su parte, el Programa de Estudios tiene como propósito principal, en cuanto a la asignatura de Formación Cívica y Ética se refiere, desarrollar en los alumnos los valores democráticos de manera plena para orientar su proyecto de vida y su forma de actuar para mejorar la sociedad en la que vive, contemplando el crecimiento personal y, por ende, impactar de mejor manera en el ámbito social.

De igual forma, mediante la comprensión y apreciación de los valores para la democracia, se pretende que los alumnos los pongan en práctica en su vida diaria y regular así, su participación democrática en la sociedad con un sentido de justicia (SEP, 2011). Razón por la que el presente documento constituye un referente fundamental del presente estudio.

En el Programa de la asignatura Formación Cívica y Ética se definen como competencias para la convivencia a aquellas que buscan formar a los alumnos con actitudes favorables para una buena relación, fomentando el trabajo colaborativo, la toma de acuerdos y el respeto a la diversidad; y las competencias para la vida en sociedad las que esperan desarrollar en los alumnos el actuar con juicio crítico ante los valores y normas de la sociedad, partiendo desde los valores democráticos y los derechos humanos. El desarrollo de estas competencias se debe dar de manera gradual y progresiva, partiendo desde el ámbito personal de los alumnos hasta llegar a los temas sobre la convivencia en el contexto social (SEP, 2011).

Con respecto a la orientación metodológica de esta asignatura, el enfoque didáctico contempla que el trabajo que los docentes realizan sobre la Formación Cívica y Ética está orientado por un conjunto de ideas y principios enfocados en el desarrollo de la autonomía en el alumno y en la adquisición de compromisos ciudadanos, partiendo de los valores para la democracia y el respeto hacia las leyes y los derechos humanos.

La asignatura está compuesta por tres ejes que constituyen un área formativa y de atención a los alumnos, además de fungir como orientadores de problemas y situaciones cotidianas que relacionan los contenidos con el contexto de los alumnos. Los ejes son: Formación de la persona, Formación ética y Formación ciudadana; mismos que contribuyen a que la formación cívica y ética favorezca que los alumnos reflexionen, analicen, y acepten los retos y oportunidades que la sociedad mexicana y el mundo les presentan, para asumircompromisos al participar en acciones que les permitan convivir y actuar de manera comprometida con el mejoramiento de la vida social. (SEP, 2011, p. 171)

Con respecto a los valores, que es otro de los conceptos claves dentro de este estudio, Yarce (2004) refiere que "sirven para formar personas que aspiren a la excelencia y la vean como una meta posible, pues su fundamento no son modas pasajeras sino un estilo de vida, una filosofía del estudio y del trabajo" (p. 58). En este sentido, los valores no sólo pautan el comportamiento y acciones, sino que contribuyen a 
formar parte del estilo de vida y de la dignidad humana; dotan de sentido a la vida y guían los conocimientos y sentimientos. El hecho de vivir siguiendo los valores asegura ser personas de bien, con hábitos y comportamientos positivos que, por ende, favorecen sin duda el estilo de vida de cualquier persona.

Por otra parte, considerar los valores como parte de los Programas de Estudio implica, a su vez, educar en valores. Es decir, crear las condiciones necesarias para que los alumnos desarrollen las actitudes y valores esenciales para que su formación fortalezca el sentido de conciencia y de responsabilidad social (Alonso, 2004); por lo que no se educa únicamente en conocimientos científicos, sino que se hace de manera íntegra.

Asimismo, cuando se habla de convivencia escolar, se hace referencia al conjunto de interacciones y relaciones que se dan en una comunidad educativa. A diferencia de la convivencia que se da fuera del aula, en la sociedad, la convivencia escolar tiene un carácter formador e impulsor de los valores y actitudes necesarias para formar a los futuros ciudadanos de la sociedad; además de ser parte fundamental del proceso de aprendizaje de los alumnos. Al respecto, Correa (2005) asegura que la buena armonía, la convivencia, el respeto y la disciplina escolar son elementos necesarios para conseguir los fines y objetivos de la educación y más aún dentro de los valores y actitudes sociales de la educación está la adquisición vivencial de las normas y reglas de respeto y convivencia como objetivos y contenidos transversales del sistema educativo. Ayuda a comprender que hay un orden moral en el mundo. Enseña a ser responsable y a desarrollar el autocontrol y autonomía y la convivencia. (p.165).

En este sentido, la convivencia escolar es parte fundamental del ambiente de aprendizaje que se busca propiciar en las aulas para que los alumnos puedan apropiarse de los aprendizajes esperados y desarrollar las competencias necesarias de mejor manera, mediante actividades que involucren el trabajo cooperativo, el diálogo y el respeto. Contribuyendo así el tratamiento de las competencias para la convivencia y para la vida en sociedad.

Con respecto a las teorías pedagógicas, no menos importantes en el tratamiento de este tema, sustentan la manera en la que se lleva a cabo un determinado proceso de enseñanza, así como sus estrategias e ideologías. Particularmente, la teoría socio-históricocultural de Vygotsky y el modelo de desarrollo moral de Kohlberg resultan fundamentales en la presente investigación, al centrar la mirada en la convivencia escolar.

Vygotsky plantea que el desarrollo cultural de los niños aparece en dos ocasiones, una en el entorno social y otra en lo individual. Primeramente, aprende del entorno y medio que lo rodea, para posteriormente procesarlo él mismo y así reconstruir el aprendizaje tomando en cuenta su propia cultura (Vygotsky, 1979 citado por Martínez, 1999). 
Este hecho, se puede distinguir en el contexto del presente estudio de la siguiente manera: para que los alumnos puedan interiorizar los valores democráticos y las actitudes y conductas necesarias para una buena convivencia, es necesario que interactúen en el aula con sus pares, mediante diversas estrategias que los adentre en el tema. Posteriormente, vivenciando los valores y actitudes junto con sus compañeros mediante dinámicas de grupo, podrán hacer suyos estos valores y practicarlos en lo individual. Es decir, después de un consecutivo proceso de adaptación y apropiación, los sujetos interiorizarán las conductas y valores democráticos necesarios para una sana convivencia.

Por su parte, Kohlberg plantea en su teoría que el desarrollo de la moral no se realiza de manera inconsciente ni a través de reforzamientos y castigos (característica del conductismo). Refiere que existen principios morales universales que no se aprenden, sino que se adquieren a través de la realización de juicios racionales, implicando un proceso cognitivo más maduro. Al respecto, Palomo (1989) expresa que "Los principales aspectos del desarrollo moral, según estas teorías, son universales porque surgen de la interacción social y todas las culturas tiene las mismas fuentes, los mismos conflictos sociales, la misma capacidad de asumir roles, etc.”. A su vez, Kohlberg propone la existencia de tres niveles de desarrollo moral desglosados en seis estadios, donde se incluyen valores, razones y perspectiva social que se tiene sobre la moralidad en cada uno de dichos estadios.

Para que el desarrollo moral de los niños vaya escalando en los estadios, hay dos factores que influyen: el proceso cognoscitivo del niño y la participación social que tiene en diversos grupos. Respecto a esto último, Kohlberg menciona que, para el favorecimiento del proceso cognoscitivo, el planteamiento de dilemas morales apegados al contexto del alumno, propicia una oportunidad de apropiación de la moralidad que se espera desarrollen.

Razón por la que la presentación y tratamiento de dilemas morales en la clase es una pieza clave para fomentar el desarrollo moral de los niños y lograr que se apropien de las actitudes, valores y normas necesarias para generar un ambiente donde la convivencia sea sana y armónica y que, consecuentemente, influya positivamente tanto en la vida de los alumnos como en su trabajo académico.

En cuanto al ambiente de aprendizaje ideal para el desarrollo de estos ejercicios, éste debe de ser generado a través de un clima de confianza y respeto donde las interacciones entre los alumnos y con el profesor tengan un papel importante dentro del proceso deenseñanza-aprendizaje. Con respecto a las estrategias para favorecer la convivencia escolar, Colombo (2011) manifiesta que deben de estar "basadas en: el diálogo, la palabra y la escucha; cumplimiento de las normas escolares, la conversación como herramienta fundamental, y la necesidad de implementar actividades de capacitación” (p. 93). Bajo estos principios, no se trata sólo de enseñar cómo debe ser la convivencia, sino generar las condiciones y 
motivación necesaria para que ésta se produzca entre los sujetos participantes a través de actividades significativas para ellos (Ibarrola-García e Iriarte, 2012).

\section{METODOLOGÍA}

La presente investigación es de carácter cualitativo bajo la metodología de investigación- acción, donde participa un maestro que reflexiona sobre su práctica de enseñanza, con el fin de transformarla. Al respecto, Latorre (2005) refiere la importancia de la investigación entre docentes como medio para mejorar su práctica educativa porque "proporciona una verdadera oportunidad para el autodesarrollo del personal docente. Si el objetivo de la investigación es mejorar la calidad educativa, la enseñanza, concebida como actividad investigadora, tiene pleno sentido" (p.10).

Los participantes son 27 alumnos de un grupo de 4to. grado de una escuela pública estatal de Monterrey, Nuevo León, México. El grupo está integrado por 13 hombres y 14 mujeres en edades oscilantes entre nueve y diez años. Participa, asimismo, un docente frente a grupo que cursa el octavo semestre de la Licenciatura en Educación Primaria en la Normal “Miguel F. Martínez”.

Las fases incluyen la detección y reflexión sobre la problemática, la planeación y la ejecución de acciones alternativas para mejorar la situación problemática, así como la evaluación de resultados con miras a emprender un segundo ciclo, de ser necesario. La primera etapa consistió en la recopilación de la información sobre las interacciones generadas entre los alumnos haciendo registros de observaciones, y la autoevaluación de todos los alumnos con el uso de un cuestionario haciendo uso de una escala de Likert.

Para la intervención y como parte del proyecto, se diseñaron siete sesiones de clase con el propósito de favorecer la convivencia a través de la asignación de roles en los alumnos, el trabajo por equipos y el tratamiento de los contenidos de la asignatura de Formación Cívica y Ética, haciendo énfasis en cinco valores democráticos para su implementación en el grupo. En la siguiente etapa se valoró el efecto de las estrategias didácticas implementadas con las producciones de los alumnos, sus actitudes en torno a la convivencia; así como la recuperación de evidencias obtenidas a través de un grupo de enfoque compuesto por 10 alumnos del grupo.

\section{DESARROLLO Y DISCUSIÓN}

Para dar inicio a la detección de la problemática y recopilación de la información, se hizo uso de instrumentos como el Diario del Normalista (diario de campo), el diario de los alumnos y una escala de Likert aplicada a los alumnos del grupo. Esta escala se aplicó en un momento específico de la investigación como referente para identificar la manera en la que se generaban las interacciones en el 
grupo desde la perspectiva de los alumnos, incluyendo indicadores encaminados hacia el tema de la convivencia en el grupo.

A la escala se le dio el nombre de CCE (Cuestionario para la exploración de la Convivencia Escolar). El cuestionario CCE contiene once ítems (figura 1), que incluye cuatro valores de frecuencia: nunca, pocas veces, muchas veces y siempre. Está estructurado en dos partes. En la primera (seis ítems) se busca la opinión del participante desde su punto de vista como observador; los ítems tratan sobre aspectos negativos en torno a la convivencia generada en el grupo, al cual pertenece. En la segunda parte (cinco ítems), se considera al alumno como partícipe de las acciones mencionadas; incluyen acciones positivas en las que se espera haya actuado.

Figura.1 Instrumento para la exploración de la convivencia escolar en un grupo de 4to. Grado

\begin{tabular}{|c|c|c|c|c|c|}
\hline No. & $\begin{array}{l}\text { Durante mi estancia en el grupo, con qué } \\
\text { frecuencia he presenciado: }\end{array}$ & Nunca & $\begin{array}{r}\text { Pocas } \\
\text { veces }\end{array}$ & $\begin{array}{c}\text { Muchas } \\
\text { veces }\end{array}$ & Siempre \\
\hline 1 & Discusiones & & & & \\
\hline 2 & Peleas & & & & \\
\hline 3 & Que insulten a un compañero & & & & \\
\hline 4 & Que llamen por apodos a un compañero & & & & \\
\hline 5 & Que excluyan a un compañero & & & & \\
\hline 6 & Que se burlen de un compañero & & & & \\
\hline No. & $\begin{array}{c}\text { Durante mi estancia en el grupo, con qué } \\
\text { frecuencia he realizado las siguientes acciones: }\end{array}$ & Nunca & $\begin{array}{r}\text { Pocas } \\
\text { veces }\end{array}$ & $\begin{array}{c}\text { Muchas } \\
\text { veces }\end{array}$ & Siempre \\
\hline 7 & Utilizado el diálogo para resolver un problema & & & & \\
\hline 8 & Trabajado en equipo con mis compañeros & & & & \\
\hline 9 & Apoyado a un compañero con algo que necesita & & & & \\
\hline 10 & Puesto un alto a una pelea o discusión & & & & \\
\hline 11 & Hecho una votación para resolver un problema & & & & \\
\hline
\end{tabular}

Fuente: Elaboración propia.

Desde la planeación del proyecto de intervención se buscó que las estrategias didácticas favorecieran las interacciones y convivencia entre los alumnos. Para ello, se focalizaron cinco valores democráticos propuestos desde el Programa de estudio para ser tratados desde la Formación Cívica y Ética. Estos valores son: libertad, respeto, pluralidad, dignidad y justicia.

Teniendo en cuenta estos cinco valores, se prosiguió con el diseño del proyecto de intervención llamado "Mi grupo: mi equipo". Se ha considerado una sesión introductoria. En seis se tratarán los valores ya mencionados y una más como conclusión o cierre del proyecto. En las sesiones se ha planeado incluir estrategias que fomenten el aprendizaje cooperativo y el diálogo, así como la interacción cara a cara entre 
los estudiantes y la distribución de roles entre los mismos. Asimismo, se ha planificado que en cada sesión en la que se aborden los valores, los alumnos realicen un producto donde se promueva el trabajo cooperativo, se elijan las mejores producciones y se integren en un mural a realizarse durante todo el proyecto. De esta manera, se busca favorecer la convivencia en el grupo y motivarlos para trabajar en colectivo.

Como parte de las actividades de cierre se ha proyectado conversaciones motivacionales y entrevistas con deportistas destacados de los equipos locales de la ciudad de Monterrey, del club de los “Auténticos Tigres: Categoría Mayor” el jugador de fútbol americano Luis Fernando Moreno Lozano; y de "Tigres: Femenil" a la jugadora Natalia Villarreal Pardo (categoría femenil sub20 y seleccionada nacional de México). Se planificó que los alumnos sean quienes elaboren previamente las preguntas, con la finalidad de que cuestionen lo que les gustaría conocer de los jugadores. En la sesión interactuarán con los jugadores, esperando les sea más significativo el tema de la convivencia y los valores.

\section{RESULTADOS}

La escala se aplicó en el mes de diciembre del 2018 en el grupo de $4^{\circ} \mathrm{C}$. Los alumnos no estaban familiarizados con cuestionarios tipo Likert, por lo que el maestro normalista apoyó en todo momento a los alumnos durante su aplicación, aclarando dudas en caso necesario. La aplicación fue ágil, ofreciendo a los alumnos el tiempo suficiente para que reflexionaran sobre cada ítem y pudiera responder rescatando sus experiencias en torno al tipo de convivencia diaria en y con el grupo.

Al dar revisión a los resultados arrojados en la escala, hubo una constante en los alumnos. La mayoría de ellos en la primera parte referida a la presencia de peleas, discusiones, apodos, insultos, exclusión, burlas, seleccionaron los valores de "Siempre" y "Muchas veces". Hecho que reconoce y deja evidente cómo al interior del grupo se habían generado este tipo de reacciones entre los miembros; ya que las preguntas estaban encaminadas a saber la frecuencia de experiencias negativas vividas por los alumnos.

Con respecto a la segunda parte de la escala, en la que se buscaba saber el grado de involucramiento de cada alumno ante situaciones de conflicto y que no favorecieran la convivencia, existió otra constante haciéndose presentes los valores de frecuencia "Nunca" y "Pocas veces". En esta segunda parte, los ítems buscaban saber sobre las acciones positivas que los alumnos pudieran realizar en pro de la convivencia. Con ello, se da cuenta que los alumnos poco han buscado dialogar para la solución de un problema, no han trabajado en equipo, poco o nunca han apoyado a un compañero, evitado peleas entre ellos o votado para la solución de algún conflicto. Resultados que reflejan la importancia de actuar ante estas circunstancias, implementando estrategias que posibiliten la mejor interacción entre los alumnos. 
Hechos similares fueron recuperados en el Diario del Normalista. Identificando formas negativas de actuar de los algunos alumnos, observando asimismo cómo éstas impactaban en la convivencia en el aula, afectando el ambiente de aprendizaje, cooperación y armonía, necesario para propiciar un clima de confianza en el aula; clima que, sin duda, debía servir como medio para favorecer las actividades de enseñanza. Diariamente se suscitaban situaciones problemáticas entre los estudiantes, afectando negativamente la convivencia en el aula. También se habrá de referir que las decisiones que se tomaban ante estos conflictos no siempre ayudaban a solucionar el problema de convivencia que se generaba en el grupo; ya que se circunscribía a regaños y reportes a la dirección.

Se ha de reconocer que la presencia de problemas en grupos de personas siempre ha existido y es normal debido a que todo sujeto posee ideas diferentes, mismas que no siempre pueden coincidir. Ante este hecho y dado a que el rol del docente es ser guías y mediadores del proceso de enseñanza que se lleva a cabo en las aulas, es su tarea propiciar las condiciones necesarias para favorecer una sana convivencia entre todos los integrantes del grupo. Al respecto, Algarra (2016) expresa: "Sabemos que el conflicto es inherente a las relaciones humanas. Resolverlo de manera pacífica está en el centro de la convivencia democrática puesto que esto implica la relación con la diversidad, con los distintos” (p. 212).

Con respecto a los registros del diario de los niños, gran parte de ellos incluían que no les gustaban cierto momento del día debido a que "discutían con un compañero o que los habían hecho sentir emociones negativas". Asimismo, muchos escribían como sugerencia que las actividades en equipo incrementaran. Esto último debido a que en algunas de las clases el maestro normalista comenzó a implementar actividades para trabajar en equipo, por lo que fueron siendo del agrado de los alumnos. Sin embargo, esta preferencia por los trabajos en equipos no significaba que existiera siempre una buena convivencia en el grupo ya que, de igual manera, durante estas actividades seguían surgiendo situaciones conflictivas.

Al implementar el proyecto de intervención, durante las sesiones de clase diseñadas para la misma, se promovió la reflexión y el diálogo con respecto a los temas tratados (los valores). Los alumnos participaron y externaron sus dudas, así como también aportaron experiencias propias de ellos con relación a los valores. A través del diario de clase realizado por ellos mismos, se pudieron identificar comentarios positivos de los niños sobre las sesiones trabajadas, indicando de cierta manera, la significatividad de esta. Posteriormente, al finalizar el proyecto con la entrevista grupal hacia los deportistas mencionados con anterioridad, se fortalecieron los temas tratados durante el proyecto. Esto debido a que los alumnos vincularon un referente a seguir junto con la puesta en práctica de los valores democráticos. De esta manera se buscó volver el proceso de apropiación de los contenidos trabajados, a un proceso más significativo, la interiorización de los temas. Finalmente, se realizó un grupo de enfoque con 10 alumnos del grupo (los cuales fueron seleccionados de manera aleatoria) y se conversó con respecto a sus 
experiencias durante el proyecto. Todos los alumnos expresaron que el proyecto fue de su agrado, así como también lo consideraron relevante. Lo que más les gustó de las sesiones, fue el trabajar en equipo y tratar con compañeros con los que no solían interactuar regularmente. Así como también expresaron lo significativo que fue para ellos la entrevista hacia los deportistas, incluso solicitando que se volviera a realizar en un futuro.

A través de un proceso de triangulación entre la información recopilada en los diarios de los alumnos, el diario del normalista y del grupo de enfoque realizado, se pudo identificar el como en un principio en ambos diarios, se hacía mención de conflictos entre los alumnos. Sin embargo, después de la implementación del proyecto de intervención, estas incidencias se redujeron de manera considerable, reflejándose en la ausencia de las mismas tanto en el diario del normalista como en el diario del alumno. Finalmente, se fortaleció el supuesto anterior al realizar la entrevista grupal con los alumnos y comparar la información recopilada durante la sesión con los datos recopilados en los instrumentos anteriores.

\section{CONCLUSIONES}

Sin duda, una buena convivencia escolar en el aula favorece en buena medida un ambiente de aprendizaje de cooperación y armonía, necesario para propiciar un clima de confianza entre sus participantes, propiciando con ello mejores actividades de enseñanza y aprendizaje.

Asimismo, las decisiones que se toman ante los conflictos, no siempre ayudan a solucionar el problema de convivencia que existe en el grupo; ya que se pueden resumir a regaños, sanciones y prohibiciones como forma de remediar situaciones de conflicto. Remedios que frenan y dificultan el desarrollo en los alumnos de los valores democráticos que se espera promover desde las escuelas primarias, con el ánimo de orientarlos para que trabajen sobre su proyecto de vida y su forma de actuar en relación a la mejora de la sociedad en la que vive; contemplando el crecimiento personal y, por ende, impactar de mejor manera en lo social.

Al docente de educación básica le debe quedar claro que, mediante la comprensión, apreciación y puesta en práctica de los valores para la democracia, los alumnos tendrán una mejor interacción entre sus pares; tendrán una participación más democrática en la sociedad, con más sentido de justicia y arraigo. Como guías y mediadores del proceso de enseñanza, es su tarea propiciar las mejores condiciones áulicas para construir una sana convivencia, entre todos los integrantes del grupo. La aplicación de estrategias concretas en el aula puede producir en la vida cotidiana pequeñas mejoras que poco a poco vayan creando un clima escolar positivo, de cuidado, y de cooperación entre los alumnos. 


\section{REFERENCIAS}

Alonso, J. (2004). La educación en valores en la institución escolar. México: Plaza y Valdez Editores.

Algara, A. (2016). Los acuerdos del aula. Una estrategia de convivencia para fortalecer la democracia en la escuela primaria. Ra Ximhai. 12. Recuperado de: http://www.redalyc.org/articulo.oa?id=46146811013

Colombo, G. (2011). Violencia Escolar y Convivencia Escolar: Descubriendo estrategias en la vida cotidiana escolar. Revista Argentina de Sociología, 8-9, 91-93.

Delors, J. (1996). La educación encierra un tesoro. Informe a la UNESCO de la comisión internacional sobre la educación para el siglo XXl. México: Editorial Santillana.

Ibarrola-García, S. y Iriarte, C. (2012). La Convivencia Escolar en positivo. Mediación y resolución de conflictos. Madrid: Ediciones Pirámide.

NEE (2018). La educación obligatoria en México. Informe 2018. México: INEE

Latorre, A. (2015). La investigación-acción. Conocer y cambiar la práctica educativa. Tercera Edición. México: Editorial Graó.

Martínez, Á. (1999). El enfoque sociocultural en el estudio del desarrollo y la educación.

REDIE. Revista Electrónica de Investigación Educativa, vol. 1,(1), p. 22 y 25.

Palomo, A. (1989). Laurence Kohlberg: Teoría y práctica del desarrollo moral en la escuela.

Revista Interuniv. p. 4, 10 y 47. [Fecha de consulta: 31 de enero de 2019] Disponible

en: https://dialnet.unirioja.es/descarga/articulo/117615.pdf.

SEP. (2011a). Formación Cívica y Ética. Cuarto grado. México: SEP. (2011b). Plan de Estudios 2011. Educación Básica. México: SEP. (2011c). Programa de Estudios 2011.Guía para el maestro. Educación Básica. Primaria.

Cuarto grado. México: SEP.

(2012) Lineamientos generales para la convivencia escolar en las escuelas de

educación básica públicas y particulares del estado de Nuevo León. Programa Escuela Segura. (2018). Programa Nacional de Convivencia Escolar. Guía para el docente. Educación

Primaria. Cuarto grado.

Yarce, J. (2004). Valor para vivir los valores. Barcelona: Bellaqva de Ediciones y Publicaciones, S.L. 